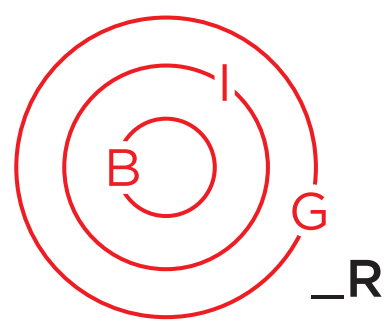

FILM REVIEW

\title{
The Land of Hope: Border, Evacuation, and Rejection During a Nuclear Crisis
}

\section{Aurélien Portelli ${ }^{\mathrm{i}}$ and Eric Rigaud ${ }^{\mathrm{ii}}$}

The Land of Hope (2012) is a Japanese film written and directed by Sion Sono. The film focuses on the consequences of setting up an evacuation perimeter following a nuclear accident in the fictitious region of "Nagashima". Sono created this name by mixing three cities: Nagasaki, Hiroshima, and Fukushima. For him, Japan has been irradiated three times. Hiroshima and Nagasaki were respectively destroyed on August 6 and 9, 1945, when the United States dropped nuclear bombs on each of the cities. Then, 66 years later on March 11, 2011, the third major nuclear event occurred at Fukushima Daiichi. During this nuclear crisis, authorities established an evacuation perimeter around the plant, leading to the displacement of neighboring populations.

Shocked by the disaster, Sono wanted to carry out a work committed to arousing public debate on nuclear power in Japan. So, for several months, he collected the testimony of many inhabitants of the Fukushima region. One particular case about a house intersected by the border delimiting the evacuation perimeter caught Sono's interest and became the inspiration for the film's script.

Before, during, or after a disaster, state authorities can evacuate a population to avoid exposure to danger. For example, in August 2005, authorities evacuated nearly one million people in New Orleans following Hurricane Katrina,

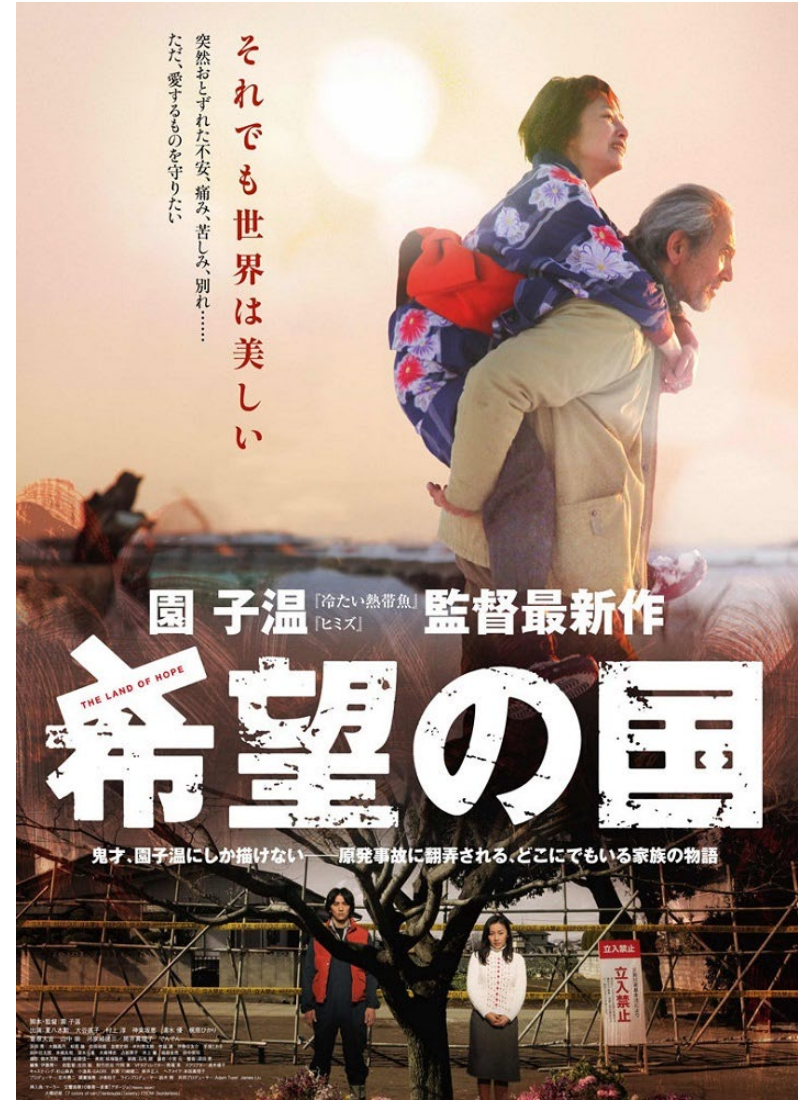

while in Fukushima nearly 160,000 people were displaced. In a nuclear accident, authorities define the evacuation area by referring to lessons learned from past events. For example, until the Fukushima accident, the standard distance of two kilometres was informed by the Three Mile Island accident from March 27, 1979. After Fukushima, the standard was expanded to 20 kilometres.

i Aurelien Portelli (PhD), Assistant Professor at the Centre of Research on Risks and Crisis, MINES Paris PSL, France. Email: aurelien.portelli@minesparis.psl.eu

ii Eric Rigaud (PhD), Assistant Professor at the Centre of Research on Risks and Crisis, MINES Paris PSL, France. Email: eric.rigaud@minesparis.psl.eu 
In The Land of Hope, authorities establish the physical location of the border between contaminated and uncontaminated spaces by estimating the distance from the damaged Nagashima plant and searching for practical elements allowing to hang the ribbon that materializes the border. The evacuation perimeter they established cut down the middle of the Ono family's garden. This physical boundary reveals the constraints of evacuating and selfevacuating individuals following a nuclear accident. How the characters in the film react illustrates the differences in the culture of nuclear risk, situational awareness, and decisionmaking, vis-à-vis the consequences of both the nuclear accident and the actions of authorities in the immediate post-accident decision making.

Sono wishes to illustrate the mismatch between the location of the border and the dangerousness of the space. Therefore, he excessively amplifies the difference between authorities' behaviors towards residents on both sides of the border. On the one hand, there is the side they consider dangerous where they urgently want residents to evacuate. In this case, they speak with a firm and injunctive tone. However, on the other hand, they consider the space on the other side of the line safe and want residents to go about their business. Therefore, they use a calm and benevolent tone to communicate.

The new border leads to the emergence of flows of people and conflicts. First, authorities forced the inhabitants of the 'contaminated' area to leave. The scene inside the bus shows violent exchanges occurring between the passengers who are agreeing to be displaced and those who wish to return to their homes. Then, in the area considered safe, the decision to leave their place of residence is left up to everyone individually. Members of the Ono family, whose house is on the right side of the border, made different decisions. Yasuhiko, the father, decides to stay with his wife, Chieko. He asks his son Yoichi to leave with his wife, Izumi, to build their own family in a safer place. Moreover, he entrusts

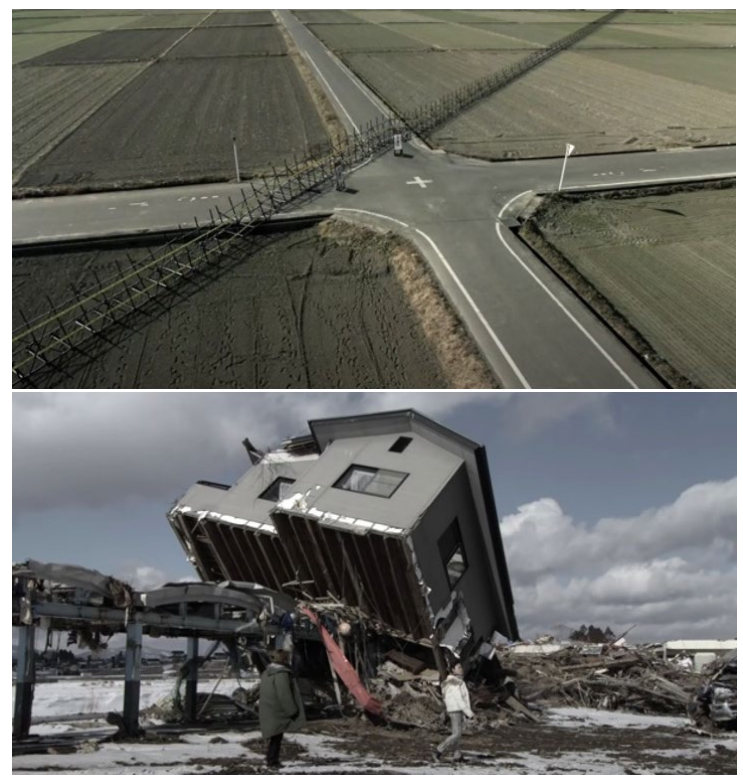

Screenshots from film trailer, images subject to copyright. his nuclear books to his daughter-in-law to help her make informed decisions. Despite multiple interventions by both the authorities and Yoichi, Yasuhiko systematically refuses to leave his farm with his wife.

Yoichi and Izumi settle in a town far from Nagashima. In one sequence, Izumi witnesses an altercation in the street. Kids brutalize a young boy coming from a contaminated area and nickname him "radioactive". In this scene, the director refers to the hibakusha, the survivors of Hiroshima and Nagasaki, who became outcasts in Japan. This rejection was due to a revulsion towards pollution deeply rooted in Japanese culture. In ancient Japan, the butchers', squarers', or tanners' descendants were sidelined from their community because they associated their activities with blood and animal death (Sabouret 2011).

Following Mary Douglas (1966), the repulsive attitude against pollution questions the relationship between order and disorder, as the film illustrates. Izumi reads the nuclear books given by her father-in-law. Obsessed with radioactivity, she begins to hallucinate. After a medical consultation, she sees behind the exit door of the hospital a red cloud, the symbol of radioactive contamination, which invades the street. An extreme close up reveals the reflection of the cloud in his eye. A blink of an eyelid dispels the hallucination, but Izumi is now possessed by fear. As the days go by, her phobia grows to the point that she has to wear a nuclear protective suit all the time. The young woman's behavior arouses incomprehension. At work, Yoichi argues with his co-workers, who feel that Izumi's outfit insults the townspeople.

According to Douglas, pollution refers to something out of place. In this respect, the radioactive particles contained in a nuclear reactor do not in themselves constitute a form of pollution. However, they become pollution after an accident when expelled from the containment and scattered outside. In response to this disorder, establishing an evacuation perimeter makes it possible to define a nuclear exclusion zone. The border is, in this case, as much of a founding act as of setting aside the world by circumscribing a territory abandoned to radioactive elements. However, the jumpsuit that Izumi wears poses a problem because it emphasizes that radioactivity is everywhere. Her attitude threatens the order instituted by the border and leads to the character's marginalization.

Thus, with The Land of Hope, Sono invites us to question the relationship between rejection, fear, and borders within the context of a nuclear crisis. The original perspective is that the displaced and the host community share the same culture and nationality.

\section{Works Cited}

Douglas, Mary. 1966. Purity and danger: An Analysis of Concepts of Pollution and Taboo. Routledge.

Sabouret, Christophe. 2011. Fukushima. L'apocalypse et après ? Pascal Galodé. 